\title{
Why Is the Immunoglobulin Heavy Chain Gene Mutation Status a Prognostic Indicator in Chronic Lymphocytic Leukemia?
}

\author{
Uri Rozovskia,b Michael J. Keating ${ }^{a} \quad$ Zeev Estrov ${ }^{a}$ \\ a Department of Leukemia, The University of Texas MD Anderson Cancer Center, Houston, TX, USA; \\ bInstitute of Hematology, Davidoff Cancer Center, Rabin Medical Center, Sackler School of Medicine, \\ Tel Aviv University, Tel Aviv, Israel
}

\section{Keywords}

Chronic lymphocytic leukemia $\cdot \lg \mathrm{HV}$ mutation status .

Somatic hypermutation

\begin{abstract}
The immunoglobulin heavy chain gene (IgHV) mutation status correlates with the clinical outcome of patients with chronic lymphocytic leukemia (CLL) treated with chemoimmunotherapy. Why the survival rate of patients with unmutated IgHV is worse than that of patients with mutated IgHV is unknown. CLL cells with unmutated IgHV were thought to originate from naïve $B$ lymphocytes, whereas CLL cells with mutated IgHV were thought to arise from B cells that have undergone somatic hypermutation (SHM). Cell surface protein expression profile and gene expression studies showing that all CLL cells, regardless of their IgHV mutation status, are of postgerminal center origin, negated this hypothesis. We hereby propose that all CLL cells undergo SHM and their proliferation rate determines their IgHV mutation status. DNA breaks, accumulated during SHM, are restored by various DNA repair mechanisms. In rapidly dividing cells DNA breaks are repaired by the efficient high-fidelity homology-directed DNA repair apparatus, whereas in slowly dividing cells they
\end{abstract}

are repaired by the inefficient low-fidelity nonhomology end-joining repair mechanism. Accordingly, a low IgHV mutation rate is found in rapidly dividing cells whereas a high mutation rate is typically found in slowly dividing cells. Thus, the proliferation rate of CLL cells determines the IgHV mutation status and patients' clinical outcome.

(C) 2018 S. Karger AG, Base

B cell chronic lymphocytic leukemia (CLL) is characterized by the accumulation of leukemic lymphocytes that coexpress CD5, CD19, and CD23. The clinical course of CLL is extremely variable. One-third of CLL patients experience an indolent disease, with a life expectancy similar to that of age-matched healthy individuals. Other patients have a benign course lasting for 5-10 years after which the disease rapidly progresses, whereas approximately $10 \%$ of patients have an aggressive disease, with a median survival of $1-3$ years from the time of diagnosis [1].

The immunoglobulin heavy chain variable region gene $(I g H V)$ mutation status has been an established prognostic factor in patients with CLL. A cutoff of $2 \%$ deviation or $98 \%$ sequence identity to germline in the $\mathrm{IgHV}$

\section{KARGER}

(c) 2018 S. Karger AG, Basel

E-Mail karger@karger.com

www.karger.com/aha
Zeev Estrov MD

Department of Leukemia, Unit 428

The University of Texas MD Anderson Cancer Center

1515 Holcombe Blvd., Houston, TX 77030 (USA)

E-Mail zestrov@mdanderson.org 
Fig. 1. The proposed model of IgHV mutation repair. a CLL cells proliferate at various rates, illustrated here by a proliferation axis. b CLL cells originating from the postgerminal center undergo SHM catalyzed by AID. A similar IgHV mutation rate (pink dots) occurs in all DNA strands irrespective of their position along the proliferation axis. c Two different mechanisms are recruited to repair the DNA breaks: the NHEJ repair, recruited by non- or slowly proliferating cells, and the homology-directed repair (HDR), recruited in rapidly proliferating cells. Because HDR is operative in rapidly proliferating cells, the higher the proliferation rate the more frequently HDR is used. d In non- or slowly proliferating CLL cells, the low-fidelity NHEJ repair mechanism does not reduce or even increases the IgHV mutation rate. Conversely, in rapidly proliferating CLL cells, the high-fidelity HDR efficiently reduces the IgHV mutation rate. e Accumulation of IgHV mutations along the proliferation axis depends on the recruitment of NHEJ vs. HDR.

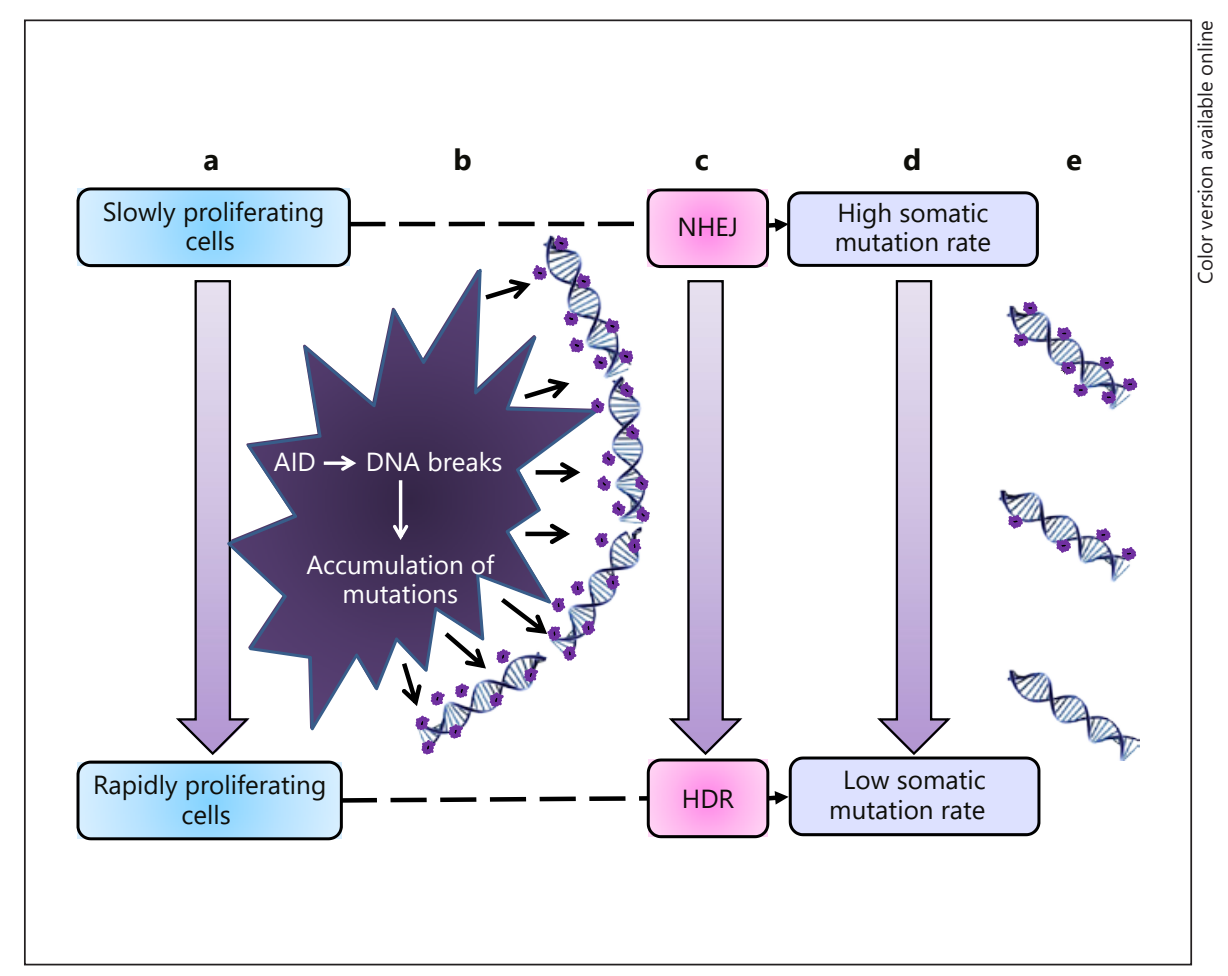

sequence has been routinely used as a prognostic marker [2]. Several studies demonstrated that patients with mutated $I g V H$ respond better to chemoimmunotherapy [3], whereas patients with unmutated $\operatorname{IgHV}$ have a shorter time to treatment, shorter time to next treatment, inferior response to chemotherapy/chemoimmunotherapy, higher chemotherapy resistance rate, and lower survival rate [4-6]. Therefore, although the significance of the $I g H V$ mutation status in patients treated with a novel agent is less clear [7], the new guidelines from the international workshop on chronic lymphocytic leukemia (iwCLL) recommend testing for $I g H V$ mutation status at baseline in all patients with CLL [8]. Although more than 20 years have passed since this observation was first reported, it is still unknown why the $I g H V$ mutation status predicts the outcome of CLL patients treated with chemoimmunotherapy.

It was proposed that CLL is not one disease but two separate entities that arise from B cells at different stages of maturation [9]. Because the genomic arrangement of CLL with unmutated $I g H V$ is similar to that of naïve B cells that undergo somatic recombination, it was postulated that $I g H V$-unmutated CLL cells arise from naïve, pregerminal center B cells and that CLL cells with mutated $\mathrm{IgHV}$ originate from postgerminal center normal B cells that underwent somatic hypermutation (SHM) after antigen recognition $[5,10]$.

However, recent data do not support this hypothesis. A retrospective analysis of the survival and progressionfree survival of 535 CLL patients treated with chemoimmunotherapy showed that the percent deviation of the immunoglobulin heavy chain gene variable region sequence from the germline sequence $(\mathrm{IgHV} \%)$ is a continuous variable. CLL patients' survival correlated with a continuous increase of $I g H V \%$ deviation. The higher the percent deviation, the better was the treatment outcome, suggesting that $I g H V \%$ is a continuous and not a dichotomized prognostic variable [11]. Furthermore, the CLL cell gene profile is independent of the $\mathrm{IgVH}$ mutation status. It is closely related to that of postgerminal center memory B cells [12] and is similar to the gene profile of CD5+ normal B cells [13]. In addition, CLL cells from all patients, regardless of the $\mathrm{IgHV}$ mutation status, express $\mathrm{CD} 27$, a surface protein that is expressed on memory B cells [14]. Also, both $I g H V$-mutated and $I g H V$-unmutated CLL cells produce a functional activation-induced deaminase (AID), an enzyme that plays a key role in SHM in normal B lymphocytes [15].

Because normal memory B cells are phenotypically similar to CLL cells and because the SHM molecular ap- 
paratus is present both in $I g H V$-mutated and $I g H V$-unmutated CLL cells, it is reasonable to assume that, similar to normal antigen-experienced memory B cells, all CLL cells undergo SHM. However, unlike in normal memory B cells, in CLL cells the IgHV gene is "mutated" in only half of the patients. In normal B cells, SHM is a 2-step process. The first step is gene transcription, during which a cytosine of single-stranded DNA undergoes deamination by AID and transforms to uracil. The second step is DNA replication, in which the DNA replication machinery recognizes uracil as thymidine and the original $\mathrm{C}: \mathrm{G}$ base pair is converted to T:A [16]. Deamination, followed by mutation, occurs frequently, even in the absence of AID, and transcription errors are usually corrected in nongerminal center $\mathrm{B}$ cells by the base excision repair mechanism using DNA polymerase I. However, the DNA of germinal center B cells is repaired by the error-prone Poln; as a result, IgHV gene mutations occur at a millionfold higher rate than spontaneous mutations in other somatic cells [16]. Because hypermutated DNA segments are fragile and tend to break [17], double-strand DNA breaks (DSBs), which accumulate during SHM near the newly formed hypermutated segments [18], activate repair mechanisms.

DSB repair mechanisms are cell cycle dependent and largely divided into 2 types: homology-directed repair and nonhomology end-joining (NHEJ) repair [19]. Homology-directed repair requires a template of a homologous chromosome and operates only in dividing cells during the S/G2 phase of the cell cycle. Homology-directed repair is a high-fidelity mechanism that restores DNA continuity and corrects DNA sequence mutations that were introduced during SHM [19]. In proliferating cells that transition through the S/G2 phase of the cell cycle, a template for homology-directed repair is readily available and constantly utilized. CLL cells that proliferate at a relatively high rate utilize this mechanism more frequently than CLL cells that proliferate at a low rate or do not proliferate at all. This high-fidelity DNA repair mechanism eliminates SHM and, as a result, the level of mutated IgHV will be low and will be defined as "unmutated." The low-fidelity NHEJ repair mechanism uses a specialized ligase to join 2 DNA ends. This mechanism does not require a template and operates throughout the cell cycle [19]. The NHEJ DNA damage-repair apparatus, recruited mainly in non- or slowly proliferating cells, does not repair DNA mutations that occur during SHM and introduces additional mutations by not correcting the loss of damaged nucleotides at the DNA break site [20]. Therefore, in non- or slowly proliferat- ing CLL cells, in which DSBs are repaired by NHEJ, the level of mutated $\mathrm{IgHV}$ will be high and defined as "mutated."

Thus, the IgHV mutation status of CLL cells is a result of a physiological recruitment of different DNA repair mechanisms during SHM. In proliferating CLL cells, high-fidelity homology-directed repair reduces $\mathrm{IgHV}$ mutations to the level of "unmutated," whereas in non- or slowly proliferating CLL cells, low-fidelity NHEJ repair does not reduce or even increases the $I g H V$ mutation rate resulting in "mutated" $\mathrm{IgHV}$, as is commonly found in normal memory B cells (Fig. 1).

Recent studies support the hypothesis that the $I g H V$ mutation status is caused by the CLL cell proliferation rate. Expanded proliferation centers, consisting of cells with a high proliferation rate, were characteristically found in lymph node biopsies of patients with unmutated but not mutated $\operatorname{IgHV}$ [21]. In addition, comprehensive heavy water labeling studies, measuring the proliferation rate of CLL cells, demonstrated a statistically significant association between increased proliferation rate and decreased IgVH mutation status [22].

We hereby propose that the IgHV gene mutation rate of CLL cells is determined by the type of the DNA repair mechanism utilized during SHM. In CLL cells that proliferated at a high rate, a high-utility repair mechanism is engaged; as a result, the $\mathrm{IgHV}$ mutation level is low (or "unmutated"). Conversely, in CLL cells that proliferated at a low rate, a low-utility repair mechanism is engaged; as a result, the $I g H V$ mutation level is high (or "mutated"). Thus, the IgHV mutation status does not directly affect the disease course or treatment outcome; it rather depends on the proliferation rate of CLL cells.

\section{Disclosure Statement}

All authors report no conflicts of interest.

References

1 Rozovski U, Hazan-Halevy I, Keating MJ, Estrov Z: Personalized medicine in CLL: current status and future perspectives. Cancer Lett 2014;352:4-14.

2 Schroeder HW Jr, Dighiero G: The pathogenesis of chronic lymphocytic leukemia: analysis of the antibody repertoire. Immunol Today $1994 ; 15: 288-294$.

3 Sagatys EM, Zhang L: Clinical and laboratory prognostic indicators in chronic lymphocytic leukemia. Cancer Control 2012;19:18-25. 
4 Damle RN, Wasil T, Fais F, Ghiotto F, Valetto A, Allen SL, Buchbinder A, Budman D, Dittmar K, Kolitz J, Lichtman SM, Schulman P, Vinciguerra VP, Rai KR, Ferrarini M, Chiorazzi $\mathrm{N}$ : Ig $\mathrm{V}$ gene mutation status and $\mathrm{CD} 38$ expression as novel prognostic indicators in chronic lymphocytic leukemia. Blood 1999; 94:1840-1847.

5 Hamblin TJ, Davis Z, Gardiner A, Oscier DG, Stevenson FK: Unmutated Ig $\mathrm{V}_{\mathrm{H}}$ genes are associated with a more aggressive form of chronic lymphocytic leukemia. Blood 1999; 94:1848-1854.

6 Lin KI, Tam CS, Keating MJ, Wierda WG, O’Brien S, Lerner S, Coombes KR, Schlette E, Ferrajoli A, Barron LL, Kipps TJ, Rassenti L, Faderl S, Kantarjian H, Abruzzo LV: Relevance of the immunoglobulin $\mathrm{VH}$ somatic mutation status in patients with chronic lymphocytic leukemia treated with fludarabine, cyclophosphamide, and rituximab (FCR) or related chemoimmunotherapy regimens. Blood 2009; 113:3168-3171.

7 Kipps TJ, Stevenson FK, Wu CJ, Croce CM, Packham G, Wierda WG, O’Brien S, Gribben J, Rai K: Chronic lymphocytic leukaemia. Nat Rev Dis Primers 2017;3:16096.

8 Hallek M, Cheson BD, Catovsky D, CaligarisCappio F, Dighiero G, Dohner H, Hillmen P, Keating M, Montserrat E, Chiorazzi N, Stilgenbauer S, Rai KR, Byrd JC, Eichhorst B, O’Brien S, Robak T, Seymour JF, Kipps TJ: iwCLL guidelines for diagnosis, indications for treatment, response assessment and supportive management of CLL. Blood 2018;131: 2745-2760.

9 Stilgenbauer S, Bullinger L, Lichter P, Dohner H; German CLL Study Group (GCLLSG): Genetics of chronic lymphocytic leukemia: genomic aberrations and $\mathrm{V}_{\mathrm{H}}$ gene mutation status in pathogenesis and clinical course. Leukemia 2002;16:993-1007.
10 Oscier DG, Thompsett A, Zhu D, Stevenson FK: Differential rates of somatic hypermutation in $\mathrm{V}_{\mathrm{H}}$ genes among subsets of chronic lymphocytic leukemia defined by chromosomal abnormalities. Blood 1997;89:41534160.

11 Jain P, Nogueras Gonzalez GM, Kanagal-Shamanna R, Rozovski U, Sarwari N, Tam C, Wierda WG, Thompson PA, Jain N, Luthra R, Quesada A, Sanchez-Petitto G, Ferrajoli A, Burger J, Kantarjian H, Cortes J, O’Brien S, Keating MJ, Estrov Z: The absolute percent deviation of IGHV mutation rather than a 98\% cut-off predicts survival of chronic lymphocytic leukaemia patients treated with fludarabine, cyclophosphamide and rituximab. Br J Haematol 2018;180:33-40.

12 Klein U, Tu Y, Stolovitzky GA, Mattioli M, Cattoretti G, Husson H, Freedman A, Inghirami G, Cro L, Baldini L, Neri A, Califano A, Dalla-Favera R: Gene expression profiling of B cell chronic lymphocytic leukemia reveals a homogeneous phenotype related to memory B cells. J Exp Med 2001;194:1625-1638.

13 Seifert M, Sellmann L, Bloehdorn J, Wein F, Stilgenbauer S, Durig J, Kuppers R: Cellular origin and pathophysiology of chronic lymphocytic leukemia. J Exp Med 2012;209: 2183-2198.

14 Damle RN, Ghiotto F, Valetto A, Albesiano E, Fais F, Yan XJ, Sison CP, Allen SL, Kolitz J, Schulman P, Vinciguerra VP, Budde P, Frey J, Rai KR, Ferrarini M, Chiorazzi N: B-cell chronic lymphocytic leukemia cells express a surface membrane phenotype of activated, antigen-experienced B lymphocytes. Blood 2002;99:4087-4093.

15 Patten PE, Chu CC, Albesiano E, Damle RN, Yan XJ, Kim D, Zhang L, Magli AR, Barrientos J, Kolitz JE, Allen SL, Rai KR, Roa S, Mongini PK, MacCarthy T, Scharff MD, Chiorazzi $\mathrm{N}$ : IGHV-unmutated and IGHV-mutated chronic lymphocytic leukemia cells produce activation-induced deaminase protein with a full range of biologic functions. Blood 2012; 120:4802-4811.
16 Liu M, Schatz DG: Balancing AID and DNA repair during somatic hypermutation. Trends Immunol 2009;30:173-181.

17 Papavasiliou FN, Schatz DG: Cell-cycle-regulated DNA double-stranded breaks in somatic hypermutation of immunoglobulin genes. Nature 2000;408:216-221.

18 Poltoratsky V, Heacock M, Kissling GE, Prasad R, Wilson SH: Mutagenesis dependent upon the combination of activation-induced deaminase expression and a double-strand break. Mol Immunol 2010;48:164-170.

19 Branzei D, Foiani M: Regulation of DNA repair throughout the cell cycle. Nat Rev Mol Cell Biol 2008;9:297-308.

20 Lieber MR: The mechanism of double-strand DNA break repair by the nonhomologous DNA end-joining pathway. Annu Rev Biochem 2010;79:181-211.

21 Gine E, Martinez A, Villamor N, Lopez-Guillermo A, Camos M, Martinez D, Esteve J, Calvo X, Muntanola A, Abrisqueta P, Rozman M, Rozman C, Bosch F, Campo E, Montserrat E: Expanded and highly active proliferation centers identify a histological subtype of chronic lymphocytic leukemia ("accelerated" chronic lymphocytic leukemia) with aggressive clinical behavior. Haematologica 2010;95:15261533.

22 van Gent R, Kater AP, Otto SA, Jaspers A, Borghans JA, Vrisekoop N, Ackermans MA, Ruiter AF, Wittebol S, Eldering E, van Oers MH, Tesselaar K, Kersten MJ, Miedema F: In vivo dynamics of stable chronic lymphocytic leukemia inversely correlate with somatic hypermutation levels and suggest no major leukemic turnover in bone marrow. Cancer Res 2008;68:10137-10144. 